\title{
Comment on the 2021 EUGOGO Clinical Practice Guidelines for the Medical Management of Graves' Orbitopathy
}

\section{Terry J Smith}

Department of Ophthalmology and Visual Sciences, and Division of Metabolism, Endocrinology and Diabetes, Department of Internal Medicine, University of Michigan Medical School, Ann Arbor, Michigan, USA

Correspondence should be addressed to T J Smith

Email

terrysmi@med.umich.edu
I am writing to you after reading the article entitled 'The 2021 European Group on Graves' Orbitopathy (EUGOGO) Clinical Practice Guidelines for the Medical Management of Graves' Orbitopathy' by Bartalena et al. (1). In these guidelines, the authors advise their colleagues who care for patients with Graves' orbitopathy (GO) to consider an aggregate of several factors in determining the optimal therapy for moderate to severe and/or sightthreatening GO. They strongly recommend as first-line therapy the combination of i.v. methylprednisolone and mycophenolate (MMF).

To be certain, a thoughtful and personalized approach to developing treatment plans is essential for consistently delivering optimal patient care. The authors of the guidelines reveal that they based their recommendations largely on clinical response parameters comprising the clinical activity score. Instead, I suggest that they should have focused on treatment effectiveness in improving the most burdensome aspects of GO, namely proptosis and diplopia. Neither steroids nor MMF has shown reliable and clinically meaningful improvements in either of these disease manifestations. Their proposed first-line therapy involves administering both agents. Their rationale for this combination appears to rest entirely on two non-placebocontrolled clinical trials. One was conducted at a single institution in China while the other was performed in Europe and failed to meet its primary response.

In contrast, teprotumumab has unambiguously demonstrated remarkable effectiveness in improving both proptosis and diplopia with relative safety in two multicenter placebo-controlled trials $(2,3)$. The US FDA has commented on the benefit-risk dimensions of teprotumumab by stating that 'Corticosteroids, orbital irradiation, and orbital surgery have been used with generally poor results'.

Thus, the authors of the guidelines should have addressed directly the skepticism expressed by the US FDA regarding the use of steroids in GO. These authors state that teprotumumab 'incorporation into routine clinical practice is currently limited by the lack of comprehensive long-term efficacy and safety data, absence of head-tohead comparison with iv glucocorticoids, restricted geographical availability, reimbursement (outside the US), and costs'. Importantly, the authors express concerns about teprotumumab because long-term efficacy and safety data are yet to be published. Yet, their recommended 'first-line' combination therapy is not supported by either long-term efficacy or safety data. Further, steroids, either as a single agent or in combination with MMF, have not achieved regulatory approval. I, therefore, urge evenhanded assessment of therapeutic options and keeping open minds regarding the prioritization of treatments, especially as teprotumumab may become commercially available in Europe for the treatment of GO.

\section{Declaration of interest}

The author was issued U.S. patents concerning the therapeutic targeting of IGF-I receptor in autoimmune diseases which are held by UCLA and Lundquist Institute. He is a paid consultant for Horizon Therapeutics.

\section{Funding}

This work did not receive any specific grant from any funding agency in the public, commercial, or not-for-profit sector. 


\section{References}

1 Bartalena L, Kahaly GJ, Baldeschi L, Dayan CM, Eckstein A, Marcocci C, Marino M, Vaidya B \& Wiersinga WM. The 2021 European Group on Graves' orbitopathy (EUGOGO) clinical practice guidelines for the medical management of Graves' orbitopathy. European Journal of Endocrinology 2021185 G43-G67. (https://doi.org/10.1530/EJE-210479)

2 Smith TJ, Kahaly GJ, Ezra DG, Fleming JC, Dailey RA, Tang RA, Harris GJ, Antonelli A, Salvi M, Goldberg RA et al. Teprotumumab for thyroid-associated ophthalmopathy. New England Journal of Medicine 2017376 1748-1761. (https://doi.org/10.1056/NEJMoa1614949)

3 Douglas RS, Kahaly GJ, Patel A, Sile S, Thompson EHZ, Perdok R, Fleming JC, Fowler BT, Marcocci C, Marino M et al. Teprotumumab for the treatment of active thyroid eye disease. New England Journal of Medicine 2020382 341-352. (https://doi.org/10.1056/NEJMoa1910434) 